\title{
Measurement and validation of energy harvesting loT devices
}

\section{Conference Paper}

Author(s):

Sigrist, Lukas (D); Gomez, Andres; Lim, Roman; Lippuner, Stefan; Leubin, Matthias; Thiele, Lothar

Publication date:

2017

Permanent link:

https://doi.org/10.3929/ethz-b-000161364

Rights / license:

In Copyright - Non-Commercial Use Permitted

Originally published in:

https://doi.org/10.23919/date.2017.7927164

Funding acknowledgement:

157048 - Transient Computing Systems (SNF) 


\title{
Measurement and Validation of Energy Harvesting IoT Devices
}

\author{
Lukas Sigrist, Andres Gomez, Roman Lim, Stefan Lippuner, Matthias Leubin, Lothar Thiele \\ Computer Engineering and Networks Laboratory (TIK), ETH Zurich, Switzerland \\ Email: \{firstname.lastname\}@ tik.ee.ethz.ch
}

\begin{abstract}
With the appearance of wearable devices and the IoT, energy harvesting nodes are becoming more and more important. The design and evaluation of these small standalone sensors and actuators, which harvest limited amounts of energy, requires novel tools and methods. Fast and accurate measurement systems are required to capture the rapidly changing harvesting scenarios and characterize leakage currents and energy efficiencies. The need for real-world experiments creates a demand for compact and portable equipment to perform in-situ power measurements and environmental logging. This work presents the RocketLogger, a hand-held measurement device that combines both properties: portability and accuracy. The custom analog front-end allows logging at sampling rates up to $64 \mathrm{kSPS}$. The fast range switching within $1.4 \mu \mathrm{s}$ guarantees continuous power measurements starting from $4 \mathrm{pW}$ at $1 \mathrm{mV}$ up to $2.75 \mathrm{~W}$ at $5.5 \mathrm{~V}$. The software provides remote control and manages data acquisition of up to $13 \mathrm{Mb} / \mathrm{sec}$ in real-time. We extensively characterize the ROCKETLOGGER's performance, demonstrate the need for its properties in three use-cases at different stages of the system design flow, and show its advantages in measuring and validating new harvesting-driven devices for the IoT.
\end{abstract}

\section{INTRODUCTION}

The advances in low power embedded systems over the past decades have considerably increased the lifetime of battery powered devices. However, deployments in hardly accessible areas and the billions of devices in the emerging Internet of Things (IoT) demand for long-term deployments with virtually unlimited lifetimes. Battery-only designs are not an option, since their limited lifetimes would require expensive maintenance. Energy harvesting and ultra-low power system design are seen as key solutions of that problem and gained increasing research attention in recent years.

Unfortunately, exploiting energy harvesting adds yet another layer of complexity to the design process. Variable and application-specific environmental conditions have a direct impact on parameters like harvested energy and power conversion efficiencies, to name a few. Harvesting-based systems need to handle a wide range of input powers from $\mathrm{nW}-\mathrm{mW}$ and adapt to changing supply conditions while efficiently using available energy. Only domain-specific solutions could meet the system's performance requirements given certain environmental constraints. The authors' experience with a broad range of such solutions like: remote sensing [1], harvesting system design [2], dimensioning [3], and management [4], and wearables [5] shows that they share a common design problem. Regardless of design strategy used, a precise characterization of the environmentdependent energy budget, characterization and optimization of the application's active and sleep currents is inevitable.

The design process of these systems typically starts with roughly estimated environmental variables like luminosity, temperature differences, vibration energy or pressure changes. Once an initial prototype is ready for testing, different metrics such as harvesting efficiency, storage element's leakage, and active/sleep currents of individual components can be measured to provide feedback to the design flow. These measurements, however, include widely ranging currents: in the $\mathrm{nA}-\mu \mathrm{A}$ range for quiescent/sleep currents, and up to 100's mA for active currents. To characterize a harvester's behavior in its production environment, as opposed to the lab, tools must be deployed with it. While measuring power and environmental properties on their own are well known problems, portable tools that accurately and reliably measure harvesting-based systems do not exist.

Without harvesting, low power systems can be validated using real-time cycle-accurate energy measurements in the lab, such as in [6]. Now system designers need to employ novel tools to design, test, and evaluate not only embedded systems themselves but also energy harvesters and the environment they operate in. There are many challenges to build these tools since they will measure a wide dynamic power range and environmental conditions in the field for potentially long periods of time. At the same time, the measurement equipment must run independently from the load being measured, influence it as little as possible, and have large memories and/or network connectivity for storing long-term measurements. Otherwise, the device will either: run out of memory quickly, not work in adverse power conditions or significantly degrade the system's harvesting or power conversion efficiency. Consequently, the measurement device must be portable, rechargeable and minimize measurement leakage currents and burden voltages. Sampling frequencies need to be high enough to capture the activity of heavily duty-cycled systems. If dynamic range switching is used, it needs to operate even faster or intermediate samples will be inaccurate. Once all the relevant measurement data has been gathered, it can be used not only to test and validate designs, but improve them in subsequent stages using iterative design methodologies.

In this paper we introduce the ROCKETLOGGER, a handheld measurement device with the diverse sensing capabilities required during harvesting-based system design. Thanks to its portable design and remote measurement manageability, it enables long-term in-situ measurements. It provides a total of four voltage and two current measurement channels with high accuracy and the ultra-high dynamic range necessary to characterize energy harvesting and application circuits. In addition, it incorporates measurements of the environmental conditions in which the system operates. The RocKeTLOGGER is the first device to balance the trade-off between a full-featured, high-performance lab measurement equipment and highly 
mobile power measurement circuits, thus satisfying essential needs of the system design process for energy harvesting driven applications.

\section{EnERgy Harvesting System Design Flow}

Through energy harvesting, sensor nodes can reach energy neutral operation. However, there are several design parameters which have a direct impact on the node's self-sustainability. For example, a transducer's harvesting and power conversion efficiencies depend on environmental conditions such as light, movement, temperature or pressure changes. In many cases, such as wearable or implantable devices, the environment is not an easily-predicted macroscopic variable. Extensive insitu measurements are thus required to gather statistically representative datasets, which can then be used in a typical embedded system design flow, as shown in Fig. 11. Several phases of the design process require diverse measurements: from environmental statistics to harvesting/conversion efficiencies and validating $\mathrm{HW} / \mathrm{SW}$ of different components or prototypes. Currently available measurement equipment does not fulfill all the requirements of these different, but related design aspects.

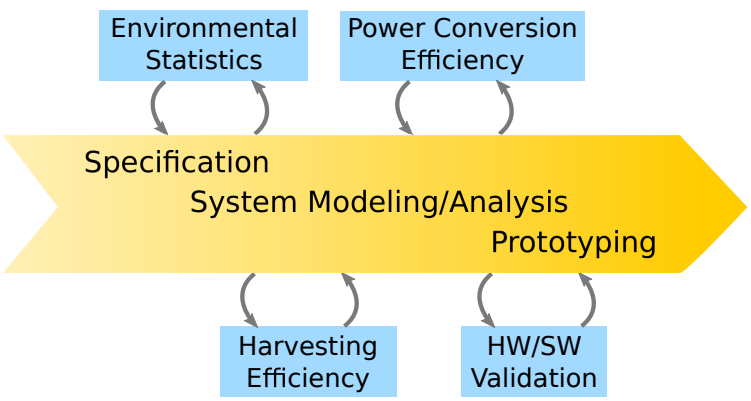

Fig. 1: Sample design aspects of harvesting-based systems. Boxes indicate aspects with distinct measurement requirements.

\section{Existing Measurement Devices}

There is a wide range of power measurement devices with varying degrees of size and functionality. On one side there are lab measurement devices that provide fast and highly accurate measurements, like the Keithley 2000 and Keysight 34400 precision digital multimeter series [7], [8]. However, these devices are bulky, require standard high voltage AC supply, and are therefore ill-suited for in-situ measurements. More recently, some specific needs of the energy harvesting based community have been addressed with new tools. These tools can be loosely classified as follows:

Online Energy Monitors: These are relatively simple measurement circuits, ranging from shunt resistors [9], [10] to coulomb counters [11], [12]. While devices can have relatively wide measurement ranges, their main design goal is to bring basic energy awareness to sensor nodes through a single current channel. Since they are tied to the load, they cannot work independently, or in adverse power conditions, and cannot provide long-term statistics.

Embedded Power Loggers: These devices are more complex than online energy monitors since they are independently powered observers with a wider dynamic range and the capability to store or forward data to a host for long-term logging. This was the approach taken by [13] and [14] to log the energy consumption of wireless embedded systems. In [15], energy traces are used in conjunction with a kernel event logger to perform detailed power analysis in smartphones. These systems, however, are designed to measure battery-based nodes with voltage regulation. In harvesting systems, designers need to measure power through independent voltage and current channels, since both are time-variant. They also require logging of environmental conditions to determine the efficiency and energy density of the harvesting circuit.

Profiling Testbeds: These platforms are also external devices which observe different parameters pertaining to one or more node. Flocklab [16], for example, can trace logical events, actuate GPIO pins, and profile the power consumption in a distributed manner. Similarly, SmartEye [17] is a testbed designed for in-situ measurements of IoT nodes, with similar capabilities but with self-sustainability in mind. The design complexities for these platforms lie either in their synchronicity or in their energy efficiency, while giving the experimenter controllable and repeatable testing of higher-level services like communication protocols. These parameters are not directly related to energy harvesting based design.

\section{System ARChitecture}

As previously discussed, measuring and validating harvesting-based systems is not a trivial task. The equipment needs to be portable, low-cost and have multiple high accuracy voltage and current channels. This requires many custom features which have to be taken into account from an early design stage. Measuring currents and voltages with such features, including seamless auto-ranging and minimal load impact, cannot be done with any commercially available system, thus requiring custom analog front-end. Different digital sensors can measure environmental variables such as temperature, illuminance and atmospheric pressure, using only a digital bus. The large volume of data, however, requires an operating system to synchronously acquire and manage the different measurement circuits and data memory. To maintain a handheld form factor, the BeagleBone Green [18] embedded Linux platform was selected. Remote user interface and data storage features were implemented on top of Linux. An overview of the architecture is shown in Fig. 2. The requirements and challenges of the implementation will now be discussed for each architecture component.

Low Current Measurement: Measuring the current generated/consumed by harvesting/application circuits is essential for early design decisions and prototyping. Low power harvesting

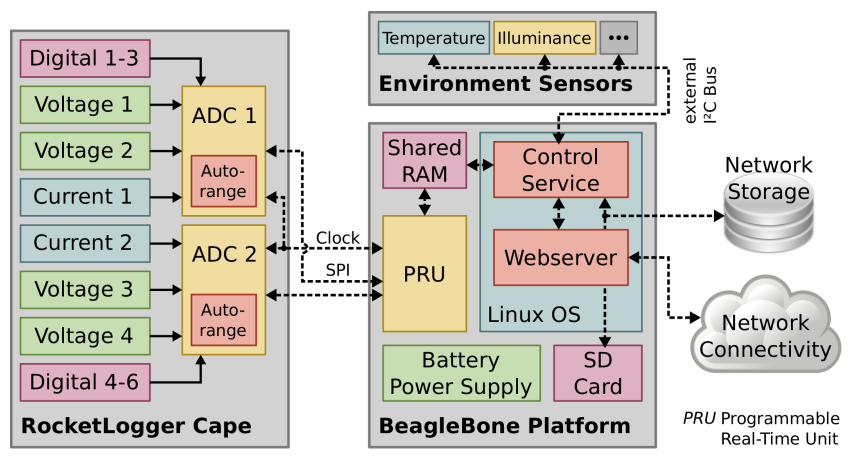

Fig. 2: Overview of the RocKETLOGGER system architecture. 


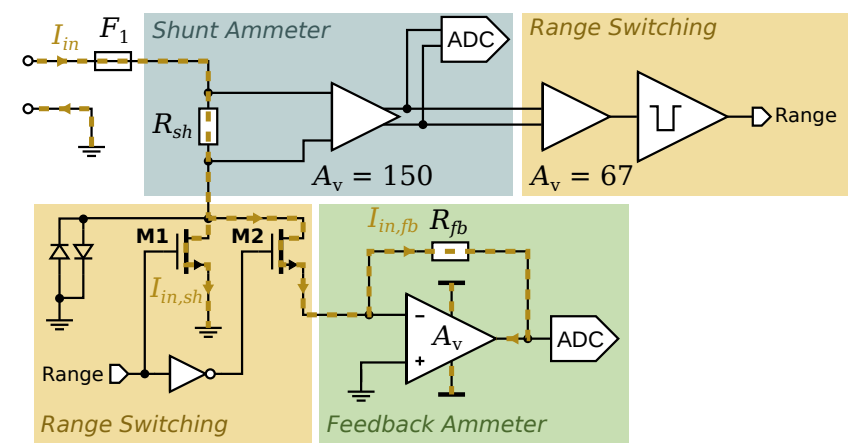

Fig. 3: High-level schematic of the RocKetLOGGER's current measurement circuitry.

scenarios and the much improved sleep current of today's microcontrollers demand current measurement ranges down to the 10's nA. To measure these low currents accurately with minimal impact on the device under test, a feedback ammeter circuit [19, Ch. 1] is used. This operational amplifier (op-amp) based circuit allows measurement of ultra-low currents in the $\mathrm{nA}$ range because of its high amplification for the feedback resistance $R_{f b}$ of $680 \Omega$. The voltage drop measured with an ADC is generated at the output of the op-amp, having the advantage of virtually zero burden voltage at the input. However, the measured current flows through the op-amp and is therefore limited by its output current capabilities. To guarantee ultra-low current measurements down to 10's nA, the RocKETLOGGER's low current measurement circuit is limited to a maximum range of $\pm 2 \mathrm{~mA}$. Beyond this range the feedback ammeter needs to be deactivated or bypassed, because it introduces an uncontrollably high burden voltage when the op-amp saturates. This is handled with the range switching circuit discussed next.

High Current Measurement: In order to measure active currents beyond $2 \mathrm{~mA}$, a traditional shunt ammeter circuit [19. Ch. 1] can be switched on. A small shunt resistance $R_{s h}$ of $50 \mathrm{~m} \Omega$ keeps the burden voltage low and minimizes the impact on the device under test. The voltage drop across the resistor is amplified with an instrumentation amplifier before analog to digital conversion. This allows accurate current measurement in the range of $2 \mathrm{~mA}$ to $500 \mathrm{~mA}$ with a noise floor in the 10 's $\mu \mathrm{A}$.

Measurement Range Switching: To get the full required measurement range of $10 \mathrm{nA}$ to $500 \mathrm{~mA}$, the RocKETLOGGER needs to switch between the two circuits. To guarantee minimal impact on the measured device during fast current transients, the range switching is integrated in the analog front-end as shown in Fig. 3. This ensures that the feedback ammeter is deactivated quickly during large current steps, e.g. a wakeup from sleep states, before it generates a large burden voltage. To detect the measurement range, an amplified version of the shunt ammeter's output signal is used: even if this signal is dominated by measurement noise for low currents, it remains valid and can be used to detect thresholds that are two orders of magnitude higher than the noise floor. The threshold detection is implemented using a window comparator. In low current range of $\leq 2 \mathrm{~mA}$ MosFET M2 is activated to pass the current also through the feedback ammeter. For higher currents the feedback ammeter is bypassed with M1 to avoid a high burden voltage in case of saturation. The shunt ammeter is always active to provide a valid input for the range switching circuit. The range signal driving the MosFETs is also used during data analysis to merge the two current measurement ranges.

Voltage Measurement: While logging the voltage is not mandatory for battery powered devices with regulated voltage supply, this becomes a necessity for energy harvesting systems. Both the harvesting and applications circuits can have timevarying operating points, which directly impacts their energy efficiency. Compared to current measurements, voltages do not cover a very large measurement range. Voltages of 10's $\mu \mathrm{V}$ up to a few volts can be measured directly using available precision ADCs. To isolate them from parallel current measurements, voltage buffers with ultra-low input leakage current are used.

Acquisition Control: Simultaneously to current and voltage measurements, 6 digital inputs are sampled for tracking the state of the system under test. These measurements are complemented by environmental logging, using temperature, luminosity, pressure, or vibration sensors. Logging the environment is essential for characterizing the harvesting sources of new designs. To control and coordinate the individual measurements a background service is running on top of the Linux operating system. While the low rate, digital environmental sensors are read out directly by this control service, the power measurement data is not. Sampling the analog and digital inputs at up to $64 \mathrm{kSPS}$ to track task executions in the sub-millisecond range results in up to $13 \mathrm{Mbs}^{-1}$ of measurement data. Because the read-out of these ADC conversion results needs to be done reactively upon data availability with very low latency, the programmable real-time unit of the BeagleBone core is used for timely transfer. This data is then buffered in RAM before it is processed by the control service. The service then combines the buffered power with the environmental samples and applies calibration before storing the measurements in the file system.

High Level Services: A data logger for harvesting driven system design is required to be portable for in-situ measurements. This in turn demands for remote control and management of data acquisition. Building on top of wired or wireless connectivity provided by the underlaying operating system, the ROCKETLOGGER exposes a web interface for remote control. It allows configuring measurement settings like sampling rate and channels to log, as well as starting and stopping the acquisition and download completed measurements. In addition, the web interface also provides online preview of running measurements. This allows checking for correct measurement setup easily and tracking of the progress during long-term data acquisitions. The network also allows offloading stored measurement data to a network drive. This is especially important for measurements longer than a few days, as local storage on the BeagleBone's $\mathrm{SD}$ card is not suitable for this purpose.

Comparison to Existing Alternatives: To summarize, the RocKETLOGGER satisfies all the requirements for long-term in-situ measurement of power and environmental conditions. It presents a well-balanced trade-off between measurement performance, portability and features. In terms of measurement performance and portability it is positioned in between high accuracy, stationary digital power meters and embedded power loggers, as the comparison of the ROCKETLOGGER with one solution of each category shows in Table I. This positions it with some unique features like integrated environmental logging that is not provided by other solutions, making the ROCKETLOGGER a perfect tool to support the design of novel energy harvesting driven devices. 
TABLE I: Positioning of the RocKetLogGer when compared to existing power logging alternatives.

\begin{tabular}{|c|c|c|c|}
\hline Characteristic & Keithley Digital Multimeter 7 & ROCKETLOGGER & Embedded Power Logger (Nemo $[9]$ ) \\
\hline Voltage Range/Accuracy & $1 \mathrm{nV}-1100 \mathrm{~V} / 0.004 \%+1.2 \mu \mathrm{V}$ & $6 \mu \mathrm{V}-5.5 \mathrm{~V} / 0.02 \%+13 \mu \mathrm{V}$ & Voltage measurement not supported \\
\hline Current Range/Accuracy & $10 \mathrm{pA}-2.1 \mathrm{~A} / 0.05 \%+1.2 \mathrm{nA}$ & $1 \mathrm{nA}-500 \mathrm{~mA} / 0.09 \%+4 \mathrm{nA}$ & $0.1 \mu \mathrm{A}-202 \mathrm{~mA} / 1.34 \%$ average, $8 \% \max$ \\
\hline Sampling Rate & up to 2 MSPS & up to $64 \mathrm{kSPS}$ & up to $100 \mathrm{kSPS}$ \\
\hline \multirow[t]{2}{*}{ Measurement Impact } & Input resistance $>100 \mathrm{G} \Omega$ & Input resistance $\sim 1 \mathrm{~T} \Omega$ & \multirow{2}{*}{ Burden voltage $\leq 130 \mathrm{mV}$} \\
\hline & Burden voltage $\leq 350 \mathrm{mV}$ & Burden voltage $\leq 53 \mathrm{mV}$ & \\
\hline Form Factor & Stationary lab device & Portable data logger & Embedded in circuit design \\
\hline Power Supply & Wall plug required & Battery powered & Powered by application circuit \\
\hline Remote Measurement & Full device control & Acquisition management and control & Not available \\
\hline Environmental Logging & Temperature only (via voltage) & $\begin{array}{l}\text { Digital sensor bus for various sensors (e.g. } \\
\text { temperature, humidity, illuminance, pressure) }\end{array}$ & Not available \\
\hline Extendability & Closed, proprietary system & Any Linux compatible program & Limited (firmware modification required) \\
\hline Cost Range/Estimate per Channel & $\$ 4900-\$ 7300$ & $\sim \$ 50$ & $\sim \$ 10$ \\
\hline
\end{tabular}

\section{Performance Characterization}

In this section the performance of the implemented measurement solution is characterized. This will show how the previously discussed requirements are met.

\section{A. Setup \& Calibration}

Before starting with performance characterization, the RocKeTLOGGER was calibrated. For the linearly designed channels, a first order calibration consisting of offset and gain is used. A Keithley 2450 source measurement unit (SMU) was configured using a stair function like input for current and voltage with steps of $2 \mathrm{~mA}$ for high current, $20 \mu \mathrm{A}$ for low current range and $100 \mathrm{mV}$ for voltage channel calibration. The calibration measurement is then analyzed in MATLAB and averaged for each current and voltage to generate the channelspecific calibration parameters.

To evaluate the performance of the logger the following three metrics are analyzed for the voltage and current channels: measurement accuracy, noise floor and bandwidth. In addition to these metrics the range switching time and burden voltage is analyzed for the current measurement circuit, as well as the input leakage current for the voltage channels. Again the SMU was used for the accuracy measurements. The noise level was characterized under zero input voltage/current conditions. For characterization of the channel bandwidth, an Agilent 33600A waveform generator generated a frequency sweep of a sine wave from $0 \mathrm{kHz}$ to $50 \mathrm{kHz}$ with amplitudes of $40 \mathrm{~mA}$ and $1 \mathrm{~V}$. Finally, the range switching time and transient burden voltage were measured for current steps from $0 \mathrm{~mA}$ to $500 \mathrm{~mA}$ using a Tektronix MSO4104B oscilloscope.

\section{B. Voltage Measurement Performance}

DC Accuracy: The accuracy of the voltage measurement was measured $24 \mathrm{~h}$ after calibration with the same voltage sweep across the full measurement range. The absolute voltage measurements are then compared to set points to analyze the DC accuracy. The results show an accuracy of $0.02 \%+13 \mu \mathrm{V}$.

Noise Floor: For characterization of the voltage channel's measurement noise, a zero input voltage experiment was used. The noise floor measurement results for $1 \mathrm{kSPS}$ show an RMS noise of $5.9 \mu \mathrm{V}$.

Bandwidth: The analog bandwidth was analyzed using a frequency sweep from $0 \mathrm{~Hz}$ up to $50 \mathrm{kHz}$. The results show that the bandwidth matches the specification of the ADC's low pass-filter: at $64 \mathrm{kSPS} \mathrm{a}-3 \mathrm{~dB}$ frequency of $10 \mathrm{kHz}$ was measured. At $1 \mathrm{kSPS}$ the bandwidth is reduced to $262 \mathrm{~Hz}$.
Input Leakage: Minimal input leakage of the voltage channels is very important when performing current measurement in parallel to not influence the current channel readings. Measurements with the power supply at the maximum input voltage of $5.5 \mathrm{~V}$ show a leakage current of around $5 \mathrm{pA}$, corresponding to an input impedance in the range of $1 \mathrm{~T} \Omega$.

\section{Current Measurement Performance}

DC Accuracy: The DC accuracy of the current channel was measured using the same setup as for the voltage channel, the only difference was to sweep the current instead of the voltage. For the current channel the DC accuracy is $0.03 \%+4 \mathrm{nA}$ for the low and $0.09 \%+3 \mu \mathrm{A}$ for the high current range.

Noise Floor: The noise floor analysis for the zero input current experiment at $1 \mathrm{kSPS}$ for low and high range show an RMS noise level of $1.33 \mathrm{nA}$ and $1.34 \mu \mathrm{A}$, respectively.

Bandwidth: The analog bandwidth was characterized with a frequency sweep using the signal generator. The $-3 \mathrm{~dB}$ frequency at $64 \mathrm{kSPS}$ is $9.5 \mathrm{kHz}$. For lower sampling rates, the bandwidth corresponds to the ones of the ADC's low-pass filter, resulting in $262 \mathrm{~Hz}$ at $1 \mathrm{kSPS}$.

Burden Voltage: The measurement of the current channel's burden voltage shows a linear behavior across the full measurement range with a maximum of $53 \mathrm{mV}$ at $500 \mathrm{~mA}$, corresponding to an impedance of $106 \mathrm{~m} \Omega$.

Range Switching Time: The experiment to characterize the current step response shows the following results: within only $1.4 \mu \mathrm{s}$ from detection, the MosFETs complete switching from low to high current range. This shows that switching in the analog front-end is one order of magnitude faster than the delay between the ADC samples at the highest sampling rate. During this short period of time, the burden voltage experiences a short peak. However, it still stays within $185 \mathrm{mV}$ for currents steps up to $100 \mathrm{~mA}$ and within $430 \mathrm{mV}$ even for the maximum current step of $500 \mathrm{~mA}$.

\section{Performance Summary}

Table III summarizes the performance characteristics of the ROCKETLOGGER, with additional characteristics for the highest sampling rate. With the shown accuracy, the very large dynamic measurement range and the portable design of only $103 \mathrm{~mm} \times 68 \mathrm{~mm}$, the ROCKETLOGGER provides trade-off between feature-rich and high precision lab equipment and fully mobile embedded measurement circuits. Its applicability throughout the design flow enables efficient design and evaluation of energy harvesting driven systems. 
TABLE II: Performance characteristics of the ROCKETLOGGER. Noise and bandwidth values shown for $1 \mathrm{kSPS}$ (64 kSPS).

\begin{tabular}{|c|c|c|}
\hline Component & Metric & Range/Value \\
\hline \multirow{3}{*}{ General } & Sampling Rate & $1 \mathrm{kSPS}$ up to $64 \mathrm{kSPS}$ \\
\hline & Data Logged & up to $8.29 \mathrm{~GB} / \mathrm{h}$ \\
\hline & Logger Size & $103 \mathrm{~mm} \times 68 \mathrm{~mm}$ \\
\hline \multirow{4}{*}{$\begin{array}{l}\text { Voltage } \\
\pm 5.5 \mathrm{~V} \\
\quad(\times 4)\end{array}$} & Noise & $5.9 \mu \mathrm{V}$ RMS (1.38 mV RMS) \\
\hline & Input Leakage & $\sim 5 \mathrm{pA}$ \\
\hline & Measurement Bandwidth & $262 \mathrm{~Hz}(10 \mathrm{kHz})$ \\
\hline & Accuracy $(24 \mathrm{~h})$ & $0.02 \%+13 \mu \mathrm{V}$ \\
\hline \multirow{10}{*}{$\begin{array}{l}\text { Current } \\
\pm 500 \mathrm{~mA} \\
\quad(\times 2)\end{array}$} & Total Dynamic Range & $172 \mathrm{~dB}$ \\
\hline & Burden Voltage at $500 \mathrm{~mA}$ & $53 \mathrm{mV}$ \\
\hline & Noise High Range & $1.34 \mu \mathrm{A}$ RMS $(50.6 \mu \mathrm{A}$ RMS $)$ \\
\hline & Low Current Range & $\pm 2 \mathrm{~mA}$ \\
\hline & Noise Low Range & $1.33 \mathrm{nA}$ RMS (276 nA RMS) \\
\hline & Range Switching Time & $1.4 \mu \mathrm{s}$ \\
\hline & Transient Burden Voltage & $\max .430 \mathrm{mV}$ for $\leq 1.4 \mu \mathrm{s}$ \\
\hline & Measurement Bandwidth & $262 \mathrm{~Hz}(9.5 \mathrm{kH} z)$ \\
\hline & Accuracy Low Range (24h) & $0.03 \%+4 \mathrm{nA}$ \\
\hline & Accuracy High Range $(24 \mathrm{~h})$ & $0.09 \%+3 \mu \mathrm{A}$ \\
\hline \multirow{2}{*}{$\begin{array}{l}\text { Digital Input } \\
(\times 6)\end{array}$} & Input Leakage & $<1 \mathrm{pA}$ \\
\hline & Threshold Voltage & configurable from $-6 \mathrm{~V}$ to $6 \mathrm{~V}$ \\
\hline
\end{tabular}

\section{Case Studies}

After focusing on the implementation and performance characterization of the ROCKETLOGGER, we will now show how its unique features enhance the design process of harvesting driven systems. To this end, three different case studies are considered: low-power duty-cycling optimizations, a multisource harvesting circuit designed for wearables, and a long-term system evaluation.

\section{A. Low Power Optimization}

In a common prototyping scenario, optimizing system sleep modes can be a trade-off between sleep power and accurate timing. This illustrates the need for ultra-low power measurement capabilities and fast, seamless range switching. In this example, we consider a $1 \mathrm{~s}$ sleep phase between execution of tasks. In this experiment an MSP430 platform was used and active tasks were simulated by turning on two LEDs. Fig. 4 shows the recorded power consumption of four different implementations of this application scenario. The right plot shows a close-up of the second task activation. Busy Idling denotes simple busy idling at the core frequency of $8 \mathrm{MHz}$ between tasks, High Freq. Timer the sleep with a timer clocked at $1 \mathrm{MHz}$ using the high frequency system clock, Low Freq. Timer again using the same timer but clocked at $9.6 \mathrm{kHz}$ from a low frequency oscillator, and Watchdog Timer where a watchdog timer, clocked by the same low frequency oscillator, is used. The plot and sleep power results show the trade-off between accurate sleep delay and ultra low power consumption of different clock sources, with the exception of the active idling which is not low power. During system prototyping, the high dynamic range of the ROCKETLOGGER is essential for evaluating and verifying different low-power design decisions and trade-offs.

\section{B. Multi-Source Wearable Harvesting}

An important phase of the harvesting circuit design is to perform real-world measurements and characterize environmental conditions, harvested power and conversion efficiencies. For wearable devices, this is particularly difficult due to the highly time-variant nature of the scenario. Furthermore, in-situ characterization demands for a portable device to be carried

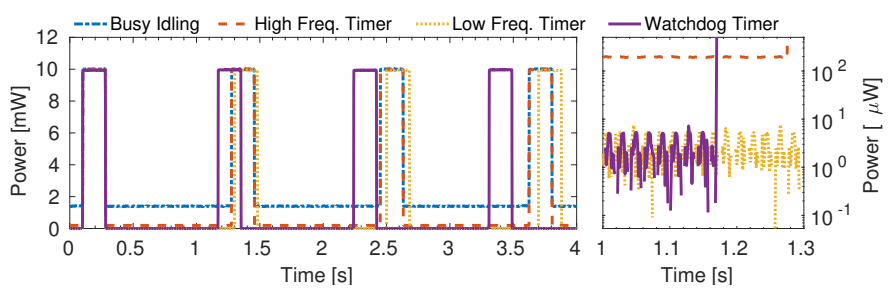

Fig. 4: Comparison of the sleep power traces for four implementation alternatives, sampled at $1 \mathrm{kSPS}$. On the right a close-up of the sleep phase of the three lowest power options is shown.

by the user. In this use case, we examine a multi-harvester wristband with solar panels and thermoelectric generators (TEG). In the test scenario shown here, the user walks outside during a warm, sunny day and then enters a colder, darker indoor space. Fig. 5] shows harvester measurements and environmental conditions, in the upper and lower plots, respectively. For the harvester measurements, it should be noted that the harvested TEG power is in the order of 100 's $\mu \mathrm{W}$, while solar power is in the $\mathrm{mW}$ range. The right $\mathrm{y}$-axis shows the operating voltages of the solar cell and the TEG. This result shows that the solar harvesting power dominates outdoors, while the TEG generates more power indoor, although at a lower power level. This data is very valuable for subsequent iterations of system modeling and analysis to optimize important system parameters like harvesting efficiency. Without independent, synchronized measurements of multiple voltage and current channels, as well as environmental conditions, this harvesting wristband could not be accurately measured or evaluated in the field.

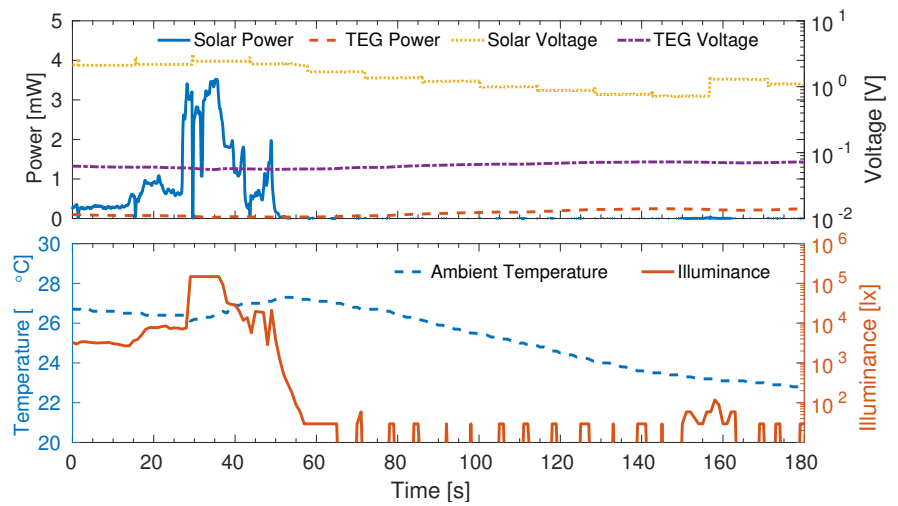

Fig. 5: Harvested power and environmental conditions of a wearable, multi-source harvester in a real-world experiment.

\section{Long-Term In-Situ System Evaluation}

One of the final prototyping stages involves testing and validating the system in the field. This requires long-term measurements to validate correct and energy efficient operation over an extended period of time. Fig. 6 shows a 7 hour slice of a day-long experiment to measure and validate a solar powered sensor node. Its harvesting circuit slowly accumulates energy in a buffer capacitor. Depending on this capacitor's voltage level, a microcontroller is triggered to perform different tasks. The node had already been tested to be functional, and was mounted on an office wall with only indirect sunlight. Fig. 6 shows the node's illuminance during the experiment in the lower plot, which closely correlates to the harvested solar power in the upper plot. It can be seen that some fault occurred during 

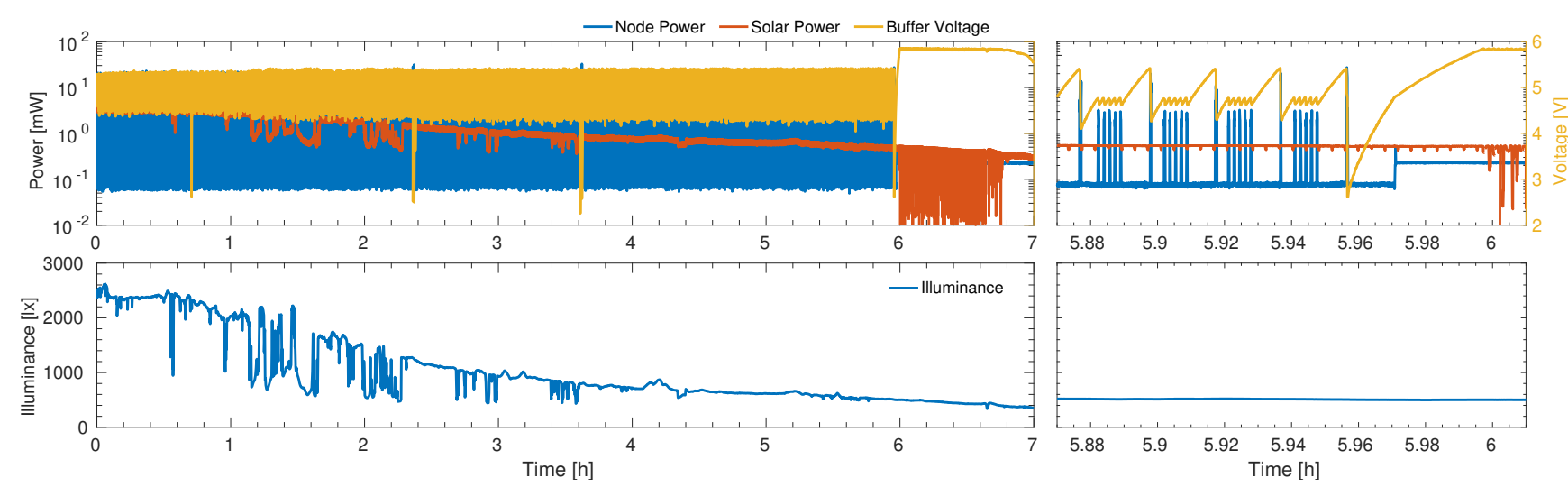

Fig. 6: Occurrence of a timing fault after $\sim 6$ hours of in-situ logging. On the right, close-ups of when the fault occurred.

the last hour of the experiment, keeping the buffer voltage high and the input power oscillating. Close-ups of when this fault happened are shown in the plots on the right. Around $t=5.97 \mathrm{~h}$, after more than 3700 successful task executions, the buffer crosses a threshold but the trace indicates that the node entered an undefined steady state. Closer investigation revealed that the triggering mechanism had, under rare and very specific circumstances, a timing fault. Device tests under standard laboratory conditions would not have revealed this fault, since it is tied to the time-varying operating points of both the source and load. Only with in-situ measurement over an extended period of time is a thorough validation of devices designed for long-term deployment possible.

\section{Discussion}

Harvesting-based system design has many different aspects which require extensive measurement data. In these casestudies, some of the main difficulties in obtaining high quality measurements have been highlighted. Traditional low power optimization, shown in case study $\mathrm{V}-\mathrm{A}$, will continue to be important and emphasizes the need for high accuracy measurements from an independent observer. Besides having multiple voltage and current channels with a wide dynamic range, measurement equipment needs to be portable and batterypowered to support in-situ measurements of mobile devices like wearables. These properties were essential to perform the measurements of case study V-B As demonstrated with case study $\mathrm{V}-\mathrm{C}$, thorough experimental validation of energy harvesting devices is very difficult without long-term in-situ measurements.

\section{CONCLUSIONS}

As energy harvesting becomes ever more prevalent in wireless sensor nodes, designers require novel tools to be able to test, validate, and characterize the performance of their designs. In this work, we have presented the RocKETLOGGER, a device capable of accurately measuring a wide dynamic power range and logging environmental conditions in long-term in-situ deployments. It combines a small shunt resistor and a feedback ammeter circuit with seamless range switching to minimize its impact on the device under test. Four voltage and two current channels are integrated in a battery-powered, hand-held measurement device. Extensive characterizations demonstrate the measurement accuracy and minimal impact on the load. Three different use-cases show how the ROCKETLOGGER's unique set of features can be used to record highly relevant and accurate measurement data. We believe this work greatly enhances the design process of future harvesting-based devices.

Acknowledgments The authors would like to thank Giovanni Rovere for his valuable input. This research was funded by the Swiss National Science Foundation under grant 157048: Transient Computing Systems.

\section{REFERENCES}

[1] J. Beutel et al., "X-SENSE: Sensing in extreme environments," in Proc. DATE Conf., 2011.

[2] C. Moser et al., "Adaptive Power Management for Environmentally Powered Systems," IEEE Trans. Comput., 2010.

[3] B. Buchli et al., "Towards enabling uninterrupted long-term operation of solar energy harvesting embedded systems," in Proc. EWSN Conf., 2014.

[4] A. Gomez et al., "Self-powered wireless sensor nodes for monitoring radioactivity in contaminated areas using unmanned aerial vehicles," in Proc. SAS, 2015.

[5] M. Magno et al., "InfiniTime: Multi-Sensor Wearable Bracelet with Human Body Harvesting," in SUSCOM, 2016.

[6] N. Chang et al., "Cycle-accurate energy consumption measurement and analysis: Case study of ARM7TDMI," in Proc. ISLPED, 2000.

[7] Keithley Instruments, "Keithley 2001/2002: $71 / 2$ - and 81/2-Digit High Performance Multimeter," accessed 8.9.2016. [Online]. Available: http:// www.tek.com/sites/tek.com/files/media/media/resources/2001-2002.pdf

[8] Keysight Technologies, "Digital Multimeters 34460A, 34461A, 34465A (61/2 digit), 34470A (71/2 digit) Data Sheet," 2016, accessed 8.9.2016. [Online]. Available: http://literature.cdn.keysight.com/litweb/ pdf/5991-1983EN.pdf

[9] R. Zhou and G. Xing, "Nemo: A high-fidelity noninvasive power meter system for wireless sensor networks," in Proc. IPSN Conf., 2013.

[10] X. Jiang et al., "Micro Power Meter for Energy Monitoring of Wireless Sensor Networks at Scale," in Proc. IPSN Conf., 2007.

[11] P. Dutta et al., "Energy Metering for Free: Augmenting Switching Regulators for Real-Time Monitoring," in Proc. IPSN Conf., 2008.

[12] S. Naderiparizi et al., " $\mu$ Monitor: In-situ Energy Monitoring with Microwatt Power Consumption," in Proc. RFID Conf., 2016.

[13] A. Pötsch et al., "A power measurement system for accurate energy profiling of embedded wireless systems," in Proc. EFTA Conf., 2014.

[14] J. Andersen and M. T. Hansen, "Energy Bucket: A Tool for Power Profiling and Debugging of Sensor Nodes," in Proc. SENSORCOMM Conf., 2009.

[15] N. Brouwers et al., "NEAT: A Novel Energy Analysis Toolkit for Freeroaming Smartphones," in Proc. SenSys Conf., 2014.

[16] R. Lim et al., "FlockLab: A testbed for distributed, synchronized tracing and profiling of wireless embedded systems," in Proc. IPSN Conf., 2013.

[17] R. Pozza et al., "SmartEye: An Energy-efficient Observer Platform for Internet of Things Testbeds," in Proc. WiNTECH Workshop, 2012.

[18] BeagleBoard.org Foundation, "SeeedStudio BeagleBone Green," accessed 1.9.2016. [Online]. Available: https://beagleboard.org/green/]

[19] Keithley Instruments, Low Level Measurements Handbook. Keithley Instruments, 2013. 